\title{
Chiral Aspects of Improved Staggered Fermions with 2+1-Flavors from the HotQCD Collaboration
}

\author{
Wolfgang Söldner* [for the HotQCD collaboration] \\ Helmholtz International Center for FAIR (HIC for FAIR), Max-von-Laue-Str. 1, D-60438 \\ Frankfurt am Main, Germany \\ E-mail: w.soeldneregsi.de
}

\begin{abstract}
We present recent results from lattice simulations of $2+1$ flavors of improved staggered fermions at zero baryon number density near the high temperature crossover. Included are new results from simulations of asqtad fermions at $N_{\tau}=12$ and a nearly physical Goldstone pion mass and from simulations of HISQ fermions at $N_{\tau}=6$ and 8 . We focus on observables sensitive to chiral symmetry and confinement. A companion HotQCD talk discusses the effects of staggered-fermion taste-symmetry breaking on thermodynamic quantities.
\end{abstract}

The XXVIII International Symposium on Lattice Field Theory, Lattice2010

June 14-19, 2010

Villasimius, Italy

\footnotetext{
*Speaker.

${ }^{\dagger}$ This work has been supported in part by contracts DE-AC02-98CH10886 and DE-FC02-06ER-41439 with the U.S. Department of Energy and contract 0555397 with the National Science Foundation. The numerical calculations have been performed using USQCD resources at Fermilab and JLab, the BlueGene/L at the New York Center for Computational Sciences (NYCCS), and the BlueGene/L at the Jülich Supercomputing Center.

${ }^{\ddagger}$ HotQCD Collaboration members are: A. Bazavov, T. Bhattacharya, M. Cheng, N.H. Christ, C. DeTar, S. Gottlieb, R. Gupta, P. Hegde, U.M. Heller, C. Jung, F. Karsch, E. Laermann, L. Levkova, C. Miao, R.D. Mawhinney, S. Mukherjee, P. Petreczky, D. Renfrew, C. Schmidt, R.A. Soltz, W. Söldner, R. Sugar, D. Toussaint, W. Unger, P. Vranas
} 

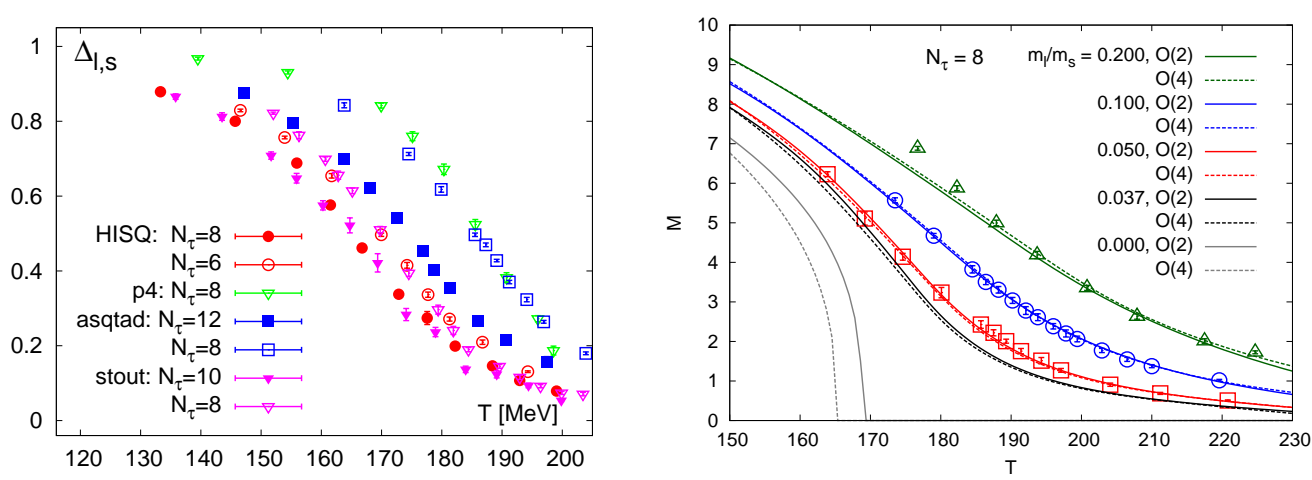

Figure 1: Left: $\Delta_{l, s}$ as a function of temperature for HISQ/tree, asqtad, and $\mathrm{p} 4$ at different $N_{\tau}$ at light quark mass $m_{l}=0.05 m_{s}$. We compare with the stout action [2]. Right: Chiral condensate $M$ for the asqtad action parameterized by the scaling function $f_{G}$.

\section{Introduction}

Understanding the properties of QCD at finite temperature is important for heavy ion collisions and the early universe. Since for the most part we need information about thermal QCD in the nonperturbative regime, lattice calculations are required. In the past couple of years we have developed a clearer understanding of the importance of cutoff effects in some thermodynamic quantities, and, as a consequence, the need for lattice calculations closer to the continuum limit and with improved actions with intrinsically smaller cutoff effects [1, 2]. Here we report on the status of ongoing calculations of the HotQCD collaboration with two light and one strange quark using the rooted staggered fermion formalism. In particular we present new results for the asqtad action at smaller lattice spacing, i.e. with temporal lattice extent $N_{\tau}=12$, and new results for HISQ [3] fermions

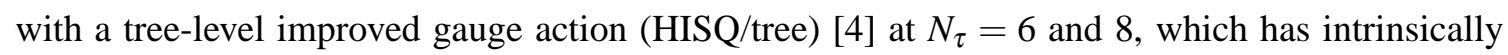
smaller cutoff effects. The strange quark mass $m_{s}$ is set to its physical value. For the light quark mass $m_{l}$ we keep the ratio $\frac{m_{l}}{m_{s}}$ fixed, resulting, approximately, in lines of constant physics over the temperature range of this study [1]. The scale is set by using $r_{1}=0.3106(8)(18)(4) \mathrm{fm}$ [5], for the calculations with HISQ/tree action we use $r_{0}=0.469 \mathrm{fm}$ to set the scale. The companion contribution [6] focuses on cutoff effects. In this contribution we investigate the chiral aspects of the finite temperature transition in 2+1-flavor QCD.

\section{Chiral aspects of the quark gluon plasma (QGP)}

The light quark chiral condensate is defined as a derivative of the QCD partition function with respect to the light quark mass, $\langle\bar{\psi} \psi\rangle_{l}=\frac{T}{V} \frac{\partial \ln Z}{\partial m_{l}}$. It is an order parameter of the QCD phase transition in the massless limit but needs a multiplicative renormalization. For finite quark mass, it also requires an additive renormalization. The combination $\Delta_{l, s}$ of light and strange quark chiral condensates at finite and zero temperature $T$ cancels the multiplicative renormalization constant and the additive renormalization constant linear in the quark mass,

$$
\Delta_{l, s}=\frac{\langle\bar{\psi} \psi\rangle_{l, T}-\frac{\hat{m}_{l}}{\hat{m}_{s}}\langle\bar{\psi} \psi\rangle_{s, T}}{\langle\bar{\psi} \psi\rangle_{l, 0}-\frac{\hat{m}_{l}}{\hat{m}_{s}}\langle\bar{\psi} \psi\rangle_{s, 0}} .
$$



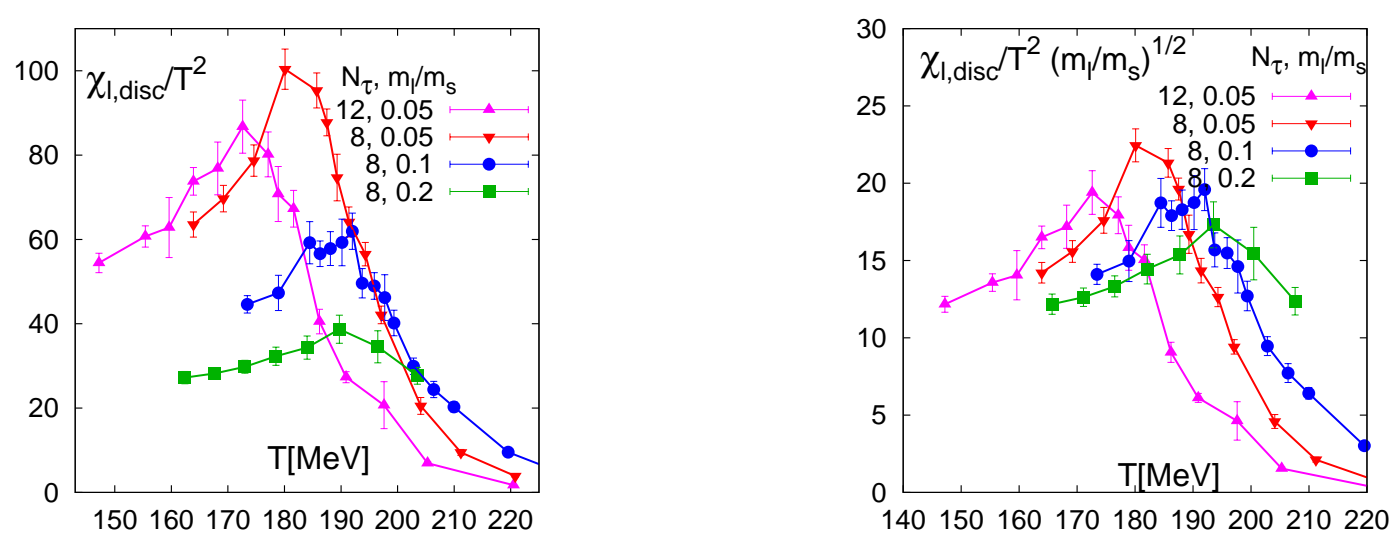

Figure 2: Disconnected chiral susceptibility for the asqtad action at different quark masses. On the right side $\chi_{1, \text { disc }}$ has been rescaled by a factor $\sqrt{m_{l} / m_{s}}$.

As can be seen on the left side of Fig. 1, the chiral condensate $\Delta_{l, s}$ drops rapidly in the transition region. As discussed in [6] we observe that cutoff effects, particularly from taste-symmetry breaking, tend to shift the transition region to higher temperatures. Those effects are less pronounced for the $\mathrm{HISQ} /$ tree action. The transition region at $m_{l}=0.05 m_{s}$ for the new $N_{\tau}=12$ asqtad data is around $T \approx 170 \mathrm{MeV}$ at this light quark mass, and for the new $N_{\tau}=8 \mathrm{HISQ} /$ tree data it is less. Let us take a closer look at the transition region. In this region physics is captured by the singular part of the partition function. For sufficiently small (light) quark mass $m_{l}$ the order parameter is described by a universal scaling function $f_{G}$ which depends on the critical exponents of the $O(2)(O(4))$ universality class, the external fields $m_{l}$ and $m_{s}$ through $H=\frac{m_{l}}{m_{s}}$, and the reduced temperature $\Delta T=\frac{T-T_{c}}{T_{c}}$, see Ref. [7] for more details.

On the right side of Fig. 1 we plot the multiplicatively renormalized chiral condensate $M_{b} \equiv$ $\frac{m_{s}}{T^{4}}\langle\bar{\psi} \psi\rangle_{l}$ and its parameterization given by the scaling function plus additional, scaling violating terms which stem from the regular part of the partition function,

$$
M_{b}\left(T, m_{l}, m_{s}\right)=h^{1 / \delta} f_{G}\left(t / h^{1 / \beta \delta}\right)+a_{t} \Delta T H+b_{1} H,
$$

where $a_{t}$ and $b_{1}$ are constants, $h=\frac{H}{h_{0}}$ and $t=\frac{\Delta T}{t_{0}}$ with scales $h_{0}$ and $t_{0}$ which need to be determined together with $T_{c}$ in the chiral limit [7]. The constants $\delta$ and $\beta$ are critical exponents. Fits based on this parameterization yield good agreement with the data in the transition region as well as for larger temperatures. Less agreement is found for larger quark masses and at lower temperatures. The latter observation may lead to the conclusion that the scaling window in the hadronic phase is significantly smaller compared to the QGP phase.

For a given lattice spacing the scaling analysis is especially useful for defining the crossover temperature $T_{p}$ and for extrapolating that temperature to its value at the physical light quark mass or at the chiral limit of zero light quark mass $\left(T_{c}\right)$. The crossover can be identified with the peak in the isosinglet chiral susceptibility, i.e., the peak in the derivative of the light quark chiral condensate with respect to the light quark mass. From the scaling analysis we know explicitly how the crossover temperature depends on the light quark mass as it approaches the critical point. We will explore this approach in more detail in a future publication. For now we investigate the critical 

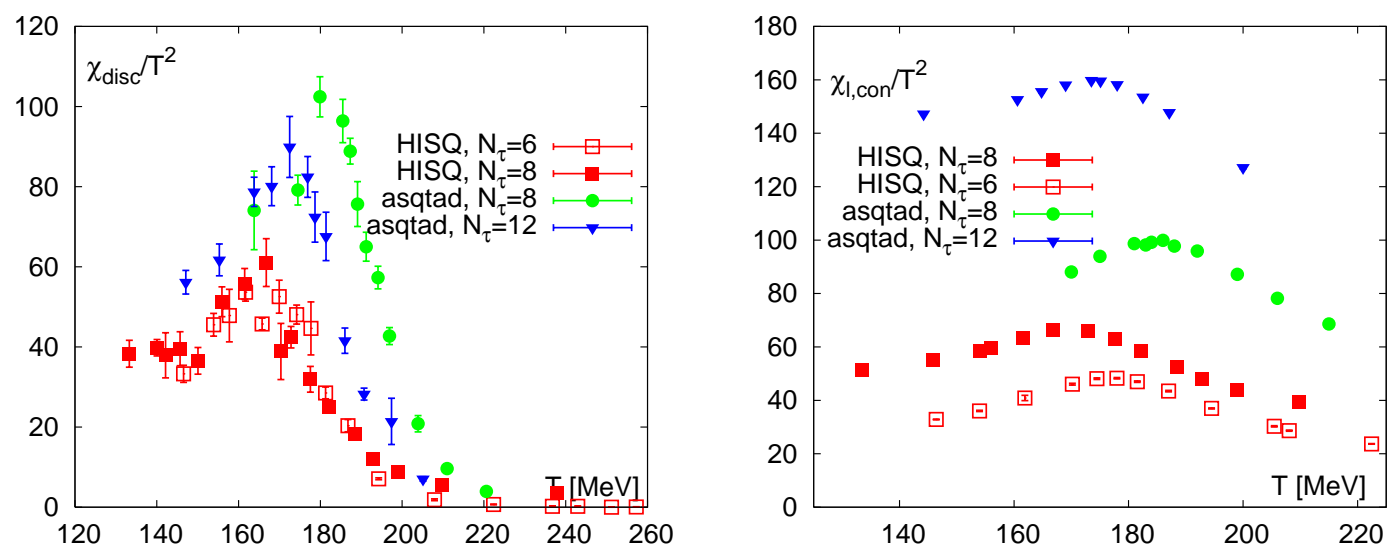

Figure 3: Left: $\chi_{1, \text { disc }}$ at fixed $m_{l}=0.05 m_{s}$ for different $N_{\tau}$ with HISQ/tree and asqtad action. Right: The connected chiral susceptibility.

temperature $T_{c}$ by studying peaks in the chiral susceptibility directly. The "full" or isosinglet chiral susceptibility $\chi_{1}^{\left(n_{f}\right)}$ in terms of the light one-flavor chiral condensate $\langle\bar{\psi} \psi\rangle_{l}$ is given by

$$
\begin{gathered}
\chi_{1}^{\left(n_{f}\right)} \equiv 2 \frac{T}{V} \frac{\partial}{\partial m_{l}}\langle\bar{\psi} \psi\rangle_{l} \equiv \chi_{1, \text { disc }}+\chi_{1, \text { con }} \quad \text { with } \\
\chi_{1, \text { disc }}=\frac{T}{4 V}\left\{\left\langle\left(\operatorname{Tr} D_{l}^{-1}\right)^{2}\right\rangle-\left\langle\operatorname{Tr} D_{l}^{-1}\right\rangle^{2}\right\} \quad \text { and } \quad \chi_{1, \text { con }} \equiv \frac{1}{2} \sum_{x}\left\langle D_{l}^{-1}(x, 0) D_{l}^{-1}(0, x)\right\rangle,
\end{gathered}
$$

where $D_{l}$ is the light quark Dirac operator and $V$ is the spatial volume. We plot the disconnected chiral susceptibility $\chi_{1, \text { disc }}$ for different $N_{\tau}$ and $m_{l}$, see left plot in Fig. 2. For all parameters the disconnected chiral susceptibility develops a clear peak structure. The height as well as the position of the peak shows a sizable quark mass dependence. On the left plot in Fig. 3 we illustrate the cutoff dependence of $\chi_{1 \text {,disc }}$. We find a mild dependence on the lattice spacing in the height and position of the peak. For the HISQ/tree action these cutoff effects are reduced compared with the asqtad action. The peak of $\chi_{1 \text {,disc }}$ at finite $N_{\tau}$ and $m_{l}$ defines pseudocritical temperatures $T_{p}$, which we use to determine the critical temperature $T_{c}$ in the chiral and continuum limit.

Before we present this analysis we consider, briefly, the quark mass dependence of $\chi_{1, \text { disc }}$. For sufficiently small quark mass the chiral condensate in the vicinity of the phase transition can be understood in terms of the three-dimensional $O(N)$ model. Below the crossover temperature and for sufficiently small light quark mass the quark mass dependence of the chiral condensate and its susceptibilities is controlled by contributions from Goldstone modes. These modes contribute a term proportional to $\sqrt{m_{l}}$ to the chiral condensate which, in turn, leads to a $1 / \sqrt{m_{l}}$ divergence in the chiral susceptibility. These features are evident in Fig. 2 .

In order to extract a continuum extrapolated critical temperature we first determine the pseudocritical temperature for several $N_{\tau}$ and $m_{l}$ by fitting the peak position of $\chi_{1 \text {,disc }}$ with different fitting ansätze where we allow for an asymmetric shape. The different ansätze allow for an estimate of systematic error in $T_{p}$. The peak location in $\chi_{1 \text {,disc }}$ is controlled by the singular part of the partition function. In the chiral limit, the disconnected chiral susceptibility $\chi_{1, \text { disc }}$ at the pseudocritical temperature exhibits critical behavior, $\chi_{1, \text { disc }}\left(T_{p}\right) \sim m_{l}^{\frac{1}{\delta}-1}$, with the critical exponent $\delta$. To extrapolate the pseudocritical temperature we use an ansatz motivated by $O(N)$ models in leading order, 


$$
T_{p}\left(m_{l}, N_{\tau}\right)=T_{c}+b\left(\frac{m_{l}}{m_{s}}\right)^{d}+c \frac{1}{N_{\tau}^{2}}
$$

where we use the critical exponent $d=$ $\frac{1}{\beta \delta} \approx 0.54$ from $\mathrm{O}(N)$ model. The last term in Eq. 2.5 accounts for the $\mathscr{O}\left(a^{2}\right)$ cutoff dependence of staggered fermions. This ansatz is supposed to work well for sufficiently small quark mass and lattice spacings. For the fitting we take into account six values of $T_{p}$ obtained with the asqtad action at $N_{\tau}=6,8,12$ at different $m_{l}$. As can be seen in Fig. 4 , the ansatz works reasonably well and we obtain a stable fit when omitting $N_{\tau}=4$ data. The black curve in this plot gives the continuum extrapolated $\left(N_{\tau} \rightarrow \infty\right)$ pseudocritical temperatures obtained from the (combined) fit. At physical mass parameters, $\frac{m_{l}}{m_{s}} \simeq \frac{1}{27}$, we then obtain a continuum extrapolated pseudocritical temperature for the chiral transition. Our preliminary estimate for the transition temperature gives,

$$
T_{p}=(164 \pm 6) \mathrm{MeV} \text {. }
$$

The error is a combined statistical and systematic estimate where also the error of the scale setting coming from $r_{1}$ is included.

The connected chiral susceptibility $\chi_{1, \text { con }}$ is an integral over the scalar, flavor nonsinglet meson correlation function, and thus is probing the thermal properties of the medium. Note that in the continuum limit $\chi_{1, \text { con }}$ can diverge in the thermodynamic limit only if the $U_{A}(1)$ symmetry is restored, which is not expected to happen at the QCD transition temperature. In fact, lattice calculations [8] indicate that the scalar screening masses develop a minimum at temperatures slightly above the transition temperature. Therefore, the connected chiral susceptibility is expected to show a maximum above the chiral transition temperature, even in the chiral limit. This is supported by our calculations by comparing the peak position of the disconnected to the connected chiral susceptibility, as shown in Fig. 3. Note that $\chi_{1, \text { con }}$ at finite quark mass suffers from additive and multiplicative renormalization. This issue will be addressed in more detail in a future publication.

\section{Deconfinement and chiral symmetry restoration}

When discussing deconfinement one often investigates quantities like the light (l) and strange (s) quark number susceptibility, $\chi_{l, s} \equiv \frac{1}{V T} \frac{\partial^{2} \ln Z}{\partial\left(\mu_{l, s} / T\right)^{2}}$, the energy density $\varepsilon$, or the Polyakov loop. Beside the Polyakov loop, where a relation (if any) to the singular part of the partition function is unknown, the other quantities are also sensitive to critical behavior. However, criticality is less pronounced in the sense that these quantities do not diverge at $T_{c}$ in the chiral limit, in contrast, e.g., to the disconnected chiral susceptibility. For example, in the chiral limit the temperature derivative of the quark number susceptibilities and the trace anomaly in the vicinity of $T_{c}$ are respectively given by,

$$
\frac{\partial \chi_{l, s}}{\partial T} \sim c_{r}^{l, s}+A_{ \pm}^{l, s}\left|\frac{T-T_{c}}{T_{c}}\right|^{-\alpha} \quad \text { and } \quad(\varepsilon-3 p) / T^{4} \sim a_{r}+b_{r} \frac{T-T_{c}}{T_{c}}+c_{ \pm}\left|\frac{T-T_{c}}{T_{c}}\right|^{1-\alpha} .
$$



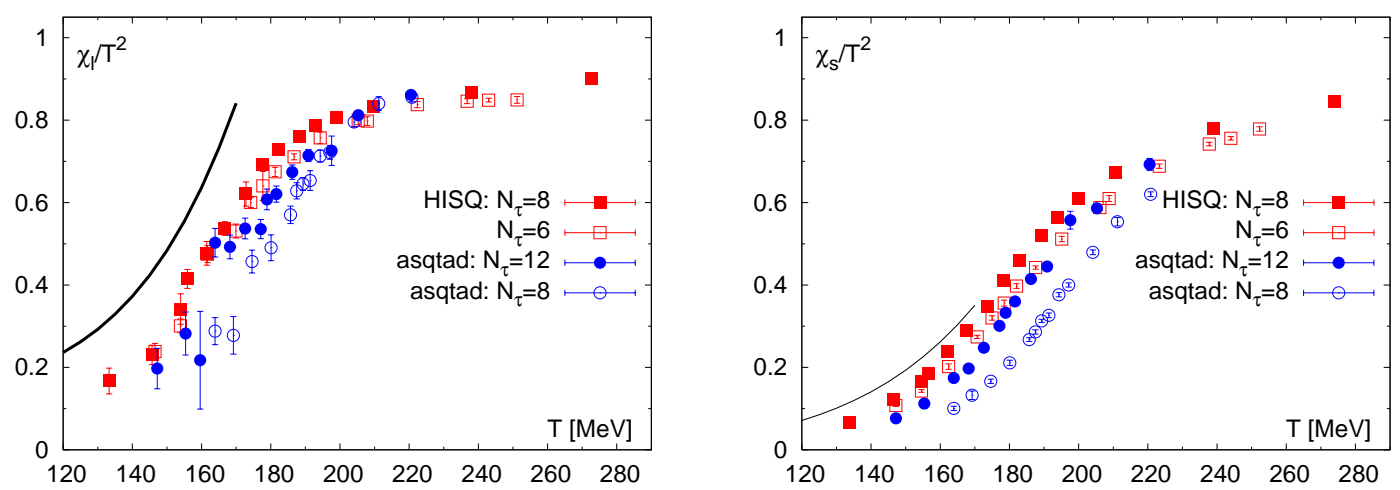

Figure 5: Light (left) and strange (right) quark number fluctuations for asqtad and HISQ/tree action compared to the HRG model (black curve).

Note that at the critical temperature $T_{c}$ the slope is controlled by a contribution that arises from the regular part of the free energy, while its variation with temperature is given by the singular part. Since the critical exponent $\alpha$ is negative it appears to be very difficult to reliably extract information about $T_{c}$ from $\chi_{l, s}$ because contributions from the regular part of the partition function may hide critical behavior. High statistics lattice data would be needed to discriminate the singular from the regular contributions. Therefore, critical behavior in state-of-the-art lattice calculations is best seen in quantities like the disconnected chiral susceptibility.

Let us take a closer look at $\chi_{l}$ and $\chi_{s}$. For low temperatures the relevant degrees of freedom which contribute to $\chi_{l, s}$ are hadrons and $\chi_{l, s}$ is small. At high temperatures the degrees of freedom are quarks and gluons. The quark number fluctuations are then related to the number of quarks and increase with temperature towards the ideal gas limit. In Fig. 5 we compare $\chi_{l}$ to $\chi_{s}$ for different $N_{\tau}$ and actions. We observe that in the vicinity of the pseudocritical temperature $\chi_{l}$ as well as $\chi_{s}$ appear to be rather smooth functions, the change in $\chi_{l}$, however, is more pronounced. In terms of the above discussion this is related to the smaller slope parameter $c_{r}^{s}$ compared to $c_{r}^{l}$. The relative magnitude of these regular terms can quite naturally be understood in terms of a hadron resonance gas (HRG) model. A more detailed investigation of this issue is currently ongoing. Note that our calculations of deconfinement and chiral quantities indicate that deconfinement and the chiral transition appear at about the same temperature, see Fig. 3 and Fig. 5 .

In Fig. 6we show the trace anomaly for different actions and quark masses. As can be seen in the left plot, at large temperatures $(\varepsilon-3 p) / T^{4}$ decreases towards zero. This is expected because at high temperatures the conformal symmetry is gradually restored. In the transition region and above $(\varepsilon-3 p) / T^{4}$ has its largest values. With respect to critical behavior the trace anomaly and the quark number susceptibilities share the same functional dependence, see Eq. 3.1. Critical behavior thus is a subdominant feature in the trace anomaly, just like in the quark number susceptibilities. Therefore, it may not be surprising that $(\varepsilon-3 p) / T^{4}$ obtains its largest value at a temperature above the pseudocritical temperature. At temperatures below the pseudocritical temperature the trace anomaly decreases and, eventually, follows the prediction of the HRG model [9]. In the plot on the right in Fig. 6 we show the low temperature region of $(\varepsilon-3 p) / T^{4}$ in more detail. Compared with our earlier calculations we observe that our new $N_{\tau}=12$ asqtad and $N_{\tau}=8 \mathrm{HISQ} /$ tree data appear to be closer to the HRG curve. However, it is also evident that at the current stage of our 

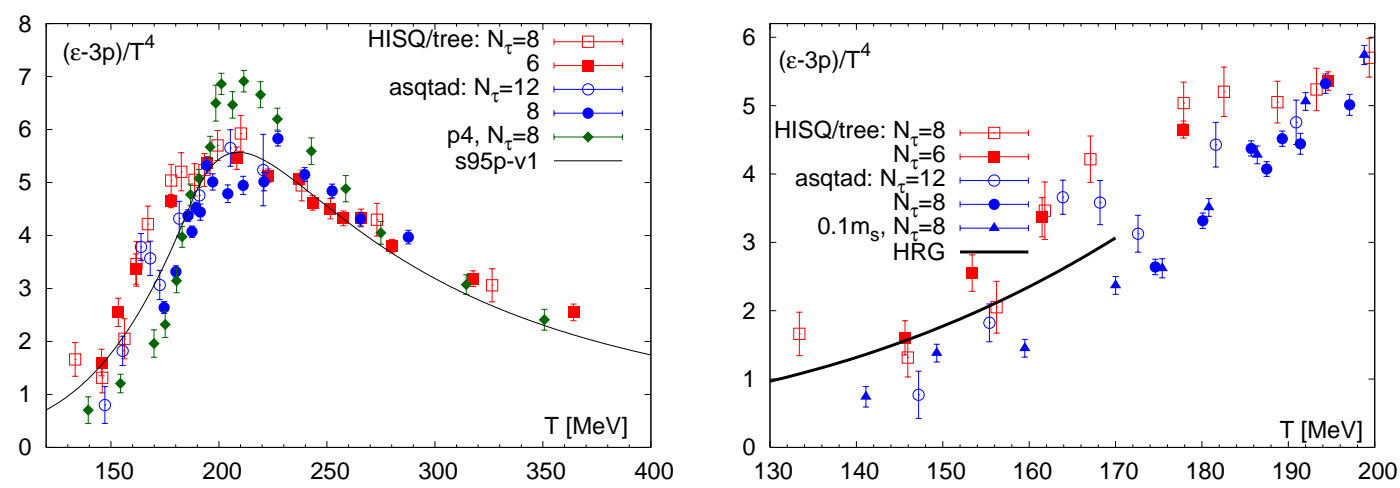

Figure 6: Left: Trace anomaly for different actions at light quark mass $m_{l}=0.05 m_{s}$ and the parameterization (black curve) from Ref. [10]. Right: Low temperature range of $(\varepsilon-3 p) / T^{4}$ at $m_{l}=0.05 m_{s}$ for HISQ/tree and asqtad action, and at $m_{l}=0.1 m_{s}$ for asqtad action at $N_{\tau}=8$. We compare to the HRG model where resonances up to $2.5 \mathrm{GeV}$ are included .

calculations the data at low temperatures is not yet conclusive and statistics for the new data sets have to be improved.

\section{Outlook}

In this contribution we have given a status report of ongoing calculations within the HotQCD collaboration focusing on chiral properties at finite temperature for QCD with $2+1$ flavors. We supplemented our earlier efforts with new calculations with asqtad fermions at $N_{\tau}=12$ and $m_{l}=$ $0.05 m_{s}$, and with HISQ/tree fermions at $N_{\tau}=6,8$ at $m_{l}=0.05 m_{s}$. With this new asqtad calculation we extracted a preliminary continuum extrapolated transition temperature at the physical point, $T_{p} \simeq(164 \pm 6) \mathrm{MeV}$. An improved determination of $T_{c}$ using universal scaling behavior is under investigation. The new HISQ/tree data in addition to upcoming HISQ/tree $N_{\tau}=12$ are much closer to the continuum limit and will enable us to further improve the continuum extrapolation. We remark that from our calculations we observe that the chiral transition and deconfinement appear at about the same temperature. Relating critical behavior to observables which are dominantly sensitive to deconfinement, i.e. the sudden change of degrees of freedom, is more subtle.

\section{References}

[1] M. Cheng et al., Phys. Rev. D 77 (2008) 014511; A. Bazavov et al., Phys. Rev. D 80 (2009) 014504.

[2] Y. Aoki et al., JHEP 0906, 088 (2009); S. Borsanyi et al., JHEP 1009, 073 (2010).

[3] E. Follana et al. [HPQCD collaboration and UKQCD collaboration], Phys. Rev. D 75, 054502 (2007).

[4] A. Bazavov et al. [HotQCD Collaboration], J. Phys. Conf. Ser. 230, 012014 (2010).

[5] C. Bernard [MILC Collaboration], PoS (LAT2010) 181.

[6] A. Bazavov et. al. [HotQCD Collaboration], PoS (LAT2010) 169.

[7] S. Ejiri et al., Phys. Rev. D 80, 094505 (2009).

[8] M. Cheng et al., arXiv:1010.1216 [hep-lat].

[9] P. Braun-Munzinger, K. Redlich and J. Stachel, arXiv:nucl-th/0304013.

[10] P. Huovinen and P. Petreczky, Nucl. Phys. A 837, 26 (2010) 